\title{
Flexive and Propulsive Dynamics of Elastica at Low Reynolds Number
}

\author{
Chris H. Wiggins ${ }^{1}$ and Raymond E. Goldstein ${ }^{2}$ \\ ${ }^{1}$ Department of Physics, Princeton University, Princeton, New Jersey 08544 \\ ${ }^{2}$ Department of Physics and Program in Applied Mathematics, University of Arizona, Tucson, Arizona 85721
}

(Received 1 August 1997)

\begin{abstract}
A stiff one-armed swimmer in glycerine goes nowhere. However, if its arm is elastic, the swimmer can go on its way. Quantifying this observation, we study a hyperdiffusion equation for the shape of the elastica in a viscous fluid, find solutions for impulsive or oscillatory forcing, and elucidate relevant aspects of propulsion. These results have application in a variety of physical and biological contexts, from dynamic experiments measuring biopolymer bending moduli to instabilities of twisted elastic filaments. [S0031-9007(98)05911-0]
\end{abstract}

PACS numbers: 87.45.-k, 03.40.Dz, 47.15.Gf

In Stokes flow, the Aristotelian fluid regime inhabited by the very small or the very slow, inertia is irrelevant. This fact underlies the inability of a variety of swimming motions, perfectly successful on human scales, to generate net motion on microscopic scales [1]. An oft quoted example is the lack of propulsion for a swimmer with only one degree of mechanical freedom, e.g., the paradigmatic scallop of Purcell's 1977 lecture Life at Low Reynolds Number which introduced many to the principles of Stokes flow [2]. Colloquially known as "the scallop theorem," this observation derives from the more general statement that motions invariant under $t \rightarrow-t$ can produce no net effect [1]; movies of Stokes flow must appear equally sensible when reversed [3].

Purcell observed two ways to elude the scallop theorem: rotate a chiral arm or wave an elastic arm. While the former dynamic is well studied (generally, in the context of E. coli [4]), the latter is largely uninvestigated, despite its relation to such experiments as motility assays and dynamic studies of biopolymer bending moduli. The notable exception is the work of Machin [5], who solved for the shape of a passive pivoting arm. After noting that the equation of motion did not support the experimentally observed wave form, Machin (and subsequent authors) instead studied the forces and flow requisite upon assuming a particular flagellar shape. Analyses in this spirit may be traced at least to 1930 [6]. We are instead interested in determining the shape via elastohydrodynamics, the balance between elasticity and viscous drag. Specifically, we study a one-armed swimmer with an elastic prosthesis, or equivalently the motion of a driven elastic filament; the passive arm produces a perfectly viable swimming motion, whose propulsive force and hydrodynamic efficiency may be calculated. Building on experiments showing the hyperdiffusivity of small-amplitude planar deformations [7], we quantify how an elastic oar eludes the scallop theorem, suggest experiments to test these results, and show how this analysis allows for measurement of bending moduli.

Force-velocity proportionalities in Stokes flow are generally not simple; notable exceptions are for highly symmetric objects such as the sphere $(F=6 \pi \mu a v$, with $\mu$ the viscosity and $a$ the radius), and those for which length $L$ greatly exceeds width $d$, where slenderbody hydrodynamics [8] applies. To lowest order in $1 / \ln (L / d)$, force and velocity obey a local, anisotropic proportionality. For velocity $v$ normal to the long axis, the force per unit length $f=\zeta_{\perp} v$, with the normal drag coefficient $\zeta_{\perp}=4 \pi \mu /[\ln (L / d)+c]$, where $c$ is an $\mathcal{O}(1)$ constant determined by the shape of the object. For an elastic filament with bending modulus $A,-f$ is the functional derivative of the bending energy $A / 2 \int_{0}^{L} d x y_{x x}^{2}$, written here for small planar deformations $y(x)$; thus, $f=-A y_{x x x x}$. At free ends the functional derivative implies boundary conditions of torquelessness and forcelessness: $y_{x x}=y_{x x x}=0$. For small deformations $v=y_{t}$, and with the hyperdiffusion constant $\tilde{\nu}=A / \zeta_{\perp}$, we have

$$
y_{t}=-\tilde{\nu} y_{x x x x},
$$

perhaps the simplest model equation of elastohydrodynamics.

In 1851, Stokes suggested two problems in fluid mechanics, here termed SI and SII (Fig. 1), to illustrate viscous diffusion of velocity [9]: SI-impulsively move a wall bounding a fluid; SII-oscillate the wall at frequency $\omega$. These motivate two problems for the elastohydrodynamic equation of motion (1): EHDI-impulsively move one end of a filament; EHDII- oscillate the end. In SI and SII, the Navier-Stokes equation reduces to a diffusion equation $u_{t}=\nu u_{x x}$, with kinematic viscosity $\nu=\mu / \rho$. For a semi-infinite domain, the post-transient solution of SII consists of decaying, right-moving waves, $u(x, t)=$ $U \exp (-\eta / \sqrt{2}) \cos (\eta / \sqrt{2}-\omega t)$, with $\eta=x / \ell_{\nu}$, and viscous penetration length $\ell_{\nu}=(\nu / \omega)^{1 / 2}$. In EHDII the analogous elastohydrodynamic penetration length is

$$
\ell_{\tilde{\nu}}(\omega)=(\tilde{\nu} / \omega)^{1 / 4} \text {. }
$$

Imposing the left filament end position $y_{0} \cos \omega t$ and torquelessness for the left end [10] $y_{x x}(0, t)=0$, we find $\frac{y}{y_{0}}=\frac{1}{2}\left[e^{-\tilde{C} \eta} \cos (\tilde{S} \eta+\omega t)+e^{-\tilde{S} \eta} \cos (\tilde{C} \eta-\omega t)\right]$, 
$\mathbf{u}$

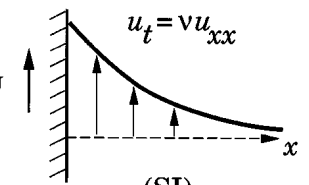

(SI)

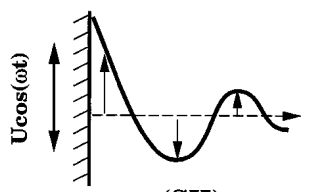

(SII)

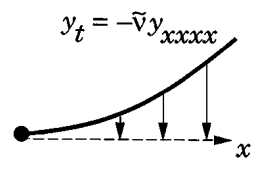

(EHDI)

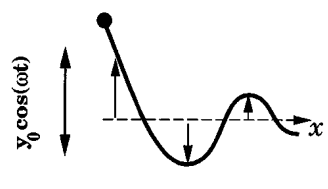

(EHDII)
FIG. 1. Geometry of Stokes problems I and II (left) and of elastohydrodynamic problems I and II (right).

where $\tilde{C}=\cos (\pi / 8), \quad \tilde{S}=\sin (\pi / 8)$, and now $\eta=$ $x / \ell_{\tilde{\nu}}$. Unlike SII, EHDII supports left- and right-moving waves (with different velocities and decay lengths), despite the lack of a reflecting right-end boundary.

For finite filaments we define a rescaled length $\mathcal{L} \equiv$ $L / \ell_{\tilde{\nu}}(\omega)$ and coordinate $\alpha=x / L$. When $\mathcal{L} \lesssim 1$ the filament behaves as a rigid rod, while it undulates appreciably for $\mathcal{L} \gg 1$. In this way $\ell_{\tilde{\nu}}$ resembles the persistence length $L_{p} \equiv A / k_{B} T$. The exact solution of EHDII for finite $L$ [11] has an expansion in powers of $\mathcal{L}^{4}$ whose first terms are

$$
\begin{aligned}
\frac{y}{y_{0}} \simeq & \left(1-\frac{3}{2} \alpha\right) \cos (\omega t)+\frac{\mathcal{L}^{4}}{1680} \\
& \times\left(16 \alpha-70 \alpha^{3}+70 \alpha^{4}-21 \alpha^{5}\right) \\
& \times \sin (\omega t)+\mathcal{O}\left(\mathcal{L}^{8}\right) .
\end{aligned}
$$

At order $\mathcal{L}^{0}$, the filament is a straight rod that pivots about a point two-thirds of its length. Flexive corrections at $\mathcal{O}\left(\mathcal{L}^{4}\right)$ break time-reversal invariance. Solutions of increasing $\mathcal{L}$ are shown in Fig. 2, whose inset shows the results of an experiment on actin [7] in which observed shapes in a range of frequencies were fit for $\ell_{\tilde{\nu}}$ to the exact expressions [11], verifying the scaling $\ell_{\tilde{\nu}} \sim \omega^{-1 / 4}$ as well as providing a novel dynamic technique for measuring the bending modulus $A$.

The propulsive force $F$ imparted to the fluid by the filament (or vice versa) may be computed by integrating the projected elastic force density along the filament. Because the (geometrically exact) elastic force per unit length can be expressed as the total derivative (with respect to arclength $s$ ) of a quantity which must vanish at free ends [11], $F$ is expressible in terms of the curvature $\kappa$ and tangent angle $\theta$ at the forcing point: $F=A\left(\kappa_{s} \sin \theta-\right.$ $\left.\frac{1}{2} \kappa^{2} \cos \theta\right)\left.\left.\right|_{s=0} \simeq A\left(y_{x x x} y_{x}-\frac{1}{2} y_{x x}^{2}\right)\right|_{x=0}$. The time average of this quantity over one period gives

$$
\bar{F}=\frac{1}{2} y_{0}^{2} \zeta_{\perp}|\omega| Y(\mathcal{L}) .
$$

For $\mathcal{L} \ll 1, Y \simeq(11 / 3360) \mathcal{L}^{4}$, so $\bar{F} \sim y_{0}^{2} \zeta_{\perp}^{2} \omega^{2} L^{4} / A$; a short (or infinitely stiff) pivoting filament produces no net force (in agreement with the scallop theorem). Flexibility leads to a net leftward propulsion, as the right-

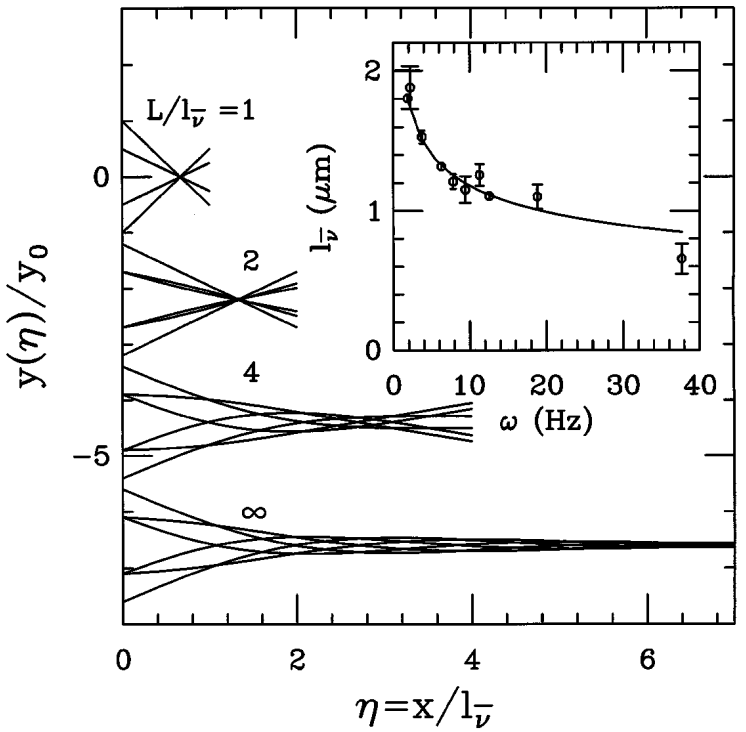

FIG. 2. Solutions to (1) for oscillatory driving at various lengths. Analogous figures for the pivoting case may be found in [5]. Inset: experimental verification [7] of the frequency dependence of $\ell_{\tilde{\nu}}(\omega)$ yielding a persistence length for actin $L_{p} \equiv A / k_{B} T=7.4 \mu \mathrm{m}$, well within the range $(0.5$ to $17 \mu \mathrm{m}$ [12]) of measurements employing statistical techniques.

moving waves dominate the left movers. An unexpected, fascinating feature is the maximum in $Y$ (Fig. 3) indicating an optimal value of the length $L_{*} \simeq 4.07 \ell_{\tilde{\nu}}$. This may be compared to studies of active or helical flagella, in which the shape is dictated and an optimal combination of parameters (e.g., helical wavelength) is chosen $[1,13]$.

In a familiar way, this force is associated with the trajectory of the filament shape in a low-dimensional

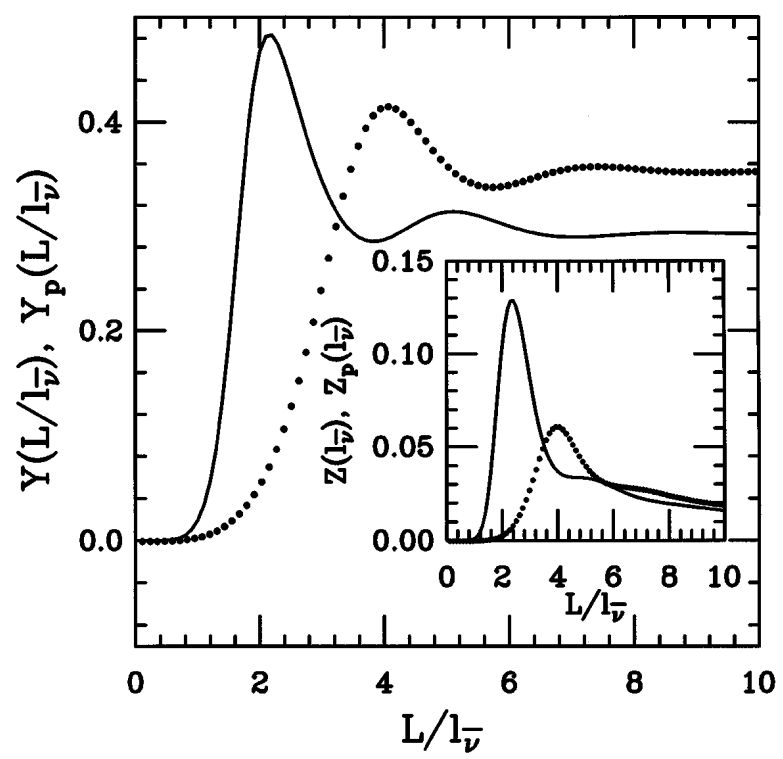

FIG. 3. Scaling functions $Y$ for propulsive force and $Z$ for efficiency versus rescaled length $\mathcal{L}=L / \ell_{\tilde{\nu}}(\omega)$. Dotted and solid lines indicate functions for EHDII or pivoting prosthesis, respectively (Inset: see text). 
projection of configuration space. The relation $F(t)=$ $A y_{x}(0, t) y_{x x x}(0, t)$ and the equation of motion imply

$\bar{F}=\frac{\zeta_{\perp} \omega}{2 \pi} \int_{0}^{2 \pi / \omega} d t y_{x} \frac{\partial}{\partial t} \int_{0}^{L} y d x \simeq \frac{\zeta_{\perp} \omega}{2 \pi} \oint \theta_{0} d \mathcal{A}$,

where $\mathcal{A}$ is the area under the curve $y(x)$ and $\theta_{0} \simeq$ $y_{x}$ is the tangent angle at the left end. Thus, the net force during the cyclic motion is the area enclosed in the associated "Carnot diagram" in $\mathcal{A}-\theta_{0}$ space; it results from pushing aside some volume of fluid (area, in two dimensions), projected via $\theta_{0}$ in the direction of propulsion. For EHDII, the trajectory is an ellipse; it thins to a straight line for the time-reversible pivoting of a rod, encloses no area, and thus produces no force. Observe the intuitive result that net propulsion in the transverse direction, proportional to $\oint d \mathcal{A}=0$, vanishes identically.

We estimate the hydrodynamic efficiency $\mathcal{E}$ of this motion [1] by comparing the power $P_{\|}=\bar{F} v_{x} \simeq \bar{F}^{2} / L \zeta_{\|}$ for longitudinal propulsion to the power $P_{\perp}=F_{y} v_{y} \simeq$ $\int d s \zeta_{\perp} y_{t}^{2}$ dissipated in transverse motions, to obtain

$$
\mathcal{E}=\frac{1}{2}\left(\frac{y_{0}}{\ell_{\tilde{\nu}}}\right)^{2} \frac{\zeta_{\perp}}{\zeta_{\|}} Z(\mathcal{L}),
$$

where $Z(\mathcal{L})$ is the scaling function shown in the inset to Fig. 3. Filaments that are short relative to $\ell_{\tilde{\nu}}(\omega)$ flex little and produce little propulsion, while long ones have excess drag from the nearly straight regions far from the point of forcing, thus explaining the sharp maximum at $\mathcal{L} \sim 4$.0. The maximum in the force may arise from a spatial resonance, brought about by the boundary conditions at the free end.

These observations suggest experiments in the spirit of those on swimming with a helical flagellum performed by Taylor [13] and later by Purcell [14]. Exploiting the results of EHDII, perhaps carried out on microfilaments with laser [7] or magnetic tweezers, or on macroscopic objects, one might measure the propulsive force through the transverse displacement at the forcing point, test for the predicted maximum as a function of frequency, investigate the role of nonlinearities, and study interactions between flexing filaments. Analogous experiments incorporating twist (perhaps via magnetic optically trapped beads, as in [15]) could investigate instabilities exhibited by the helical motion of flexible filaments [16] and associated propulsion.

The geometry described above is merely one of several reminiscent of a one-armed swimmer. Instead, one might constrain the tangent angle at the left end, similar to a pivoting arm rather than an a low-Re jump rope. The conjugates to these two conditions are constrained force or torque, respectively. To reveal the generic features of the above results we comment on these variants.

In constraining the tangent, we demand $y_{x}(0)=$ $\theta_{0} \cos (\omega t) ; y(0)=0$. Solutions for constrained torque $\left[\tau_{0} \cos (\omega t)=A y_{x x}(0)\right]$ or force $\left[F_{0} \cos (\omega t)=A y_{x x x}(0)\right]$ are obtained from those with constrained tangent and position, respectively. We may then construct the expansions for $y_{\text {force }}, y_{\text {torque }}$, and $y_{\text {pivot }}$ analogous to Eq. (4):

$$
\begin{aligned}
\frac{y_{\text {force }}}{F_{0} L^{3} / A} \simeq & \left(\frac{1}{105}-\frac{11}{216} \alpha+\frac{\alpha^{3}}{6}-\frac{\alpha^{4}}{6}+\frac{\alpha^{5}}{20}\right) \cos (\omega t) \\
& +\mathcal{L}^{-4}(4-6 \alpha) \sin (\omega t)+\mathcal{O}\left(\mathcal{L}^{4}\right) \\
\frac{y_{\text {torque }}}{T_{0} L^{2} / A} \simeq & \left(-\frac{33}{140} \alpha+\frac{\alpha^{2}}{2}-\frac{\alpha^{3}}{3}+\frac{\alpha^{5}}{40}\right) \cos (\omega t) \\
& -\mathcal{L}^{-4} \alpha \sin (\omega t)+\mathcal{O}\left(\mathcal{L}^{4}\right), \\
\frac{y_{\text {pivot }}}{\theta_{0} L}= & \alpha \cos (\omega t)+\frac{\mathcal{L}^{4}}{120}\left(\alpha^{5}+20 \alpha^{2}-10 \alpha^{3}\right) \\
& \times \sin (\omega t)+\mathcal{O}\left(\mathcal{L}^{8}\right) .
\end{aligned}
$$

Note that in the limit $\mathcal{L} \rightarrow 0$, if we constrain torque or force, we face divergent $y_{x}(0) \sim \mathcal{L}^{-3}$ or $\sim \mathcal{L}^{-2}$, respectively, leaving the linearized regime. The pivoting case, however, remains well defined in this limit.

As above, we may construct the appropriate force and efficiency diagrams as functions of $\mathcal{L}$ for the pivoting case. Averaging $\left.A\left(y_{x x x} y_{x}-\frac{1}{2} y_{x x}^{2}\right)\right|_{x=0}$ over one period, we find that the existence of a local maximum in force and efficiency is preserved (cf. Fig. 3). For real spermatazoans, the "excess" length performs work, consuming adenosine triphosphate and exerting a bending moment; as may be expected, their lengths typically exceed $L_{*}$. As an example, Lythechinus (sea urchin) spermatozoa, for which $L \simeq 38 \mu \mathrm{m}$ and $\omega=180 \mathrm{~Hz}$, have $L_{*} / \ell_{\tilde{\nu}} \sim 7.4$ [17].

The pivoting force and efficiency may be written as

$$
\begin{aligned}
& \bar{F}_{\text {pivot }}=\frac{1}{2}\left(\theta_{0} \ell_{\tilde{\nu}}\right)^{2} \zeta_{\perp}|\omega| Y_{p}(\mathcal{L}), \\
& \mathcal{E}_{\text {pivot }}=\frac{1}{2} \theta_{0}^{2} \frac{\zeta_{\perp}}{\zeta_{\|}} Z_{p}(\mathcal{L}),
\end{aligned}
$$

facilitating comparison of the propulsion for the two motions; for $\theta_{0}$ comparable to $y_{0} / \ell_{\tilde{\nu}}$, we may simply compare the dimensionless scaling functions. Each of these has a greater maximum in the latter case, with a shorter optimal length for the pivoting prosthesis: $L_{*} \simeq$ $2.2 \ell_{\tilde{\nu}}$. The peak force and efficiency are then $\sim 6 \mathrm{pN}$ (taking $\omega$ from Lythechinus and assuming $\theta_{0} \sim 0.5$ ) and 0.03 , respectively.

The appropriate Carnot diagram is slightly complicated by the nonzero $\kappa \simeq y_{x x}$ at the left end. We must add to Eq. (6) the term

$$
-\frac{\zeta_{\perp} \omega}{2 \pi} \int_{0}^{2 \pi / \omega} d t \kappa_{0}(t) \frac{d}{d t} I(t)=-\frac{\zeta_{\perp} \omega}{2 \pi} \oint \kappa_{0} d I,
$$

and $\quad I(t) \equiv 1 / 2 \int_{0}^{L} d x \mathcal{A}(x, t)=1 / 2 \int_{0}^{L} d x \times$ $\int_{0}^{x} d x^{\prime} y\left(x^{\prime}, t\right)$. Since the curvature is of order $\mathcal{L}^{4}$, this term decays even more rapidly as $\mathcal{L} \rightarrow 0$ than the term in Eq. (6). The time-averaged force is the difference of the areas enclosed by the trajectories in the $\mathcal{A}-\theta_{0}$ and $I-\kappa_{0}$ planes. 
The above analysis describes the post-transient dynamic, ignoring the decay of initial conditions. This second analysis, appropriate to either impulsive driving (EHDI) or the decay of initial data, suggests a second elastohydrodynamic technique for measuring bending moduli. Since Eq. (1) is linear, we may subtract from its general solution a particular solution, consistent with nonzero boundary conditions or external driving, to obtain a homogeneous equation with 0 -valued boundary conditions. This motivates the construction of a self-adjoint operator from $\mathcal{H} \equiv \partial_{x}^{4}$, whose well-known eigenfunctions [18] are

$$
\begin{aligned}
\mathcal{W}_{q}(x)= & a_{1} \sin (q x)+a_{2} \cos (q x) \\
& +a_{3} \sinh (q x)+a_{4} \cosh (q x),
\end{aligned}
$$

where $\mathcal{H} \mathcal{W}_{q}=q^{4} \mathcal{W}_{q}$. The ten distinct choices of boundary conditions for which $\mathcal{H}$ is self-adjoint [11] determine the distinct coefficients $\left\{a_{i}\right\}$ of $\mathcal{W}_{q}$ and the allowed wave numbers $q$. These $q$ are roots of transcendental solvability conditions [18]; certain boundary conditions are satisfied by Fourier modes (those for which $a_{3}=a_{4}=0$ ).

The completeness of the set $\mathcal{W}_{q}$ yields the homogeneous solution to Eq. (1),

$$
y(x, t)=\sum_{q} \mathcal{W}_{q}(x) e^{-\tilde{\nu} q^{4} t} \int_{0}^{L} d x \mathcal{W}_{q}(x) y(x, 0) .
$$

Since the modulus $A$ enters via $\tilde{\nu}$, identification of the allowed $\{q\}$ and observation of the decay times yield a measurement of the bending modulus. One recent example [19] is the relaxation of an initially bent microtubule. In this clever experiment, one end of the microtubule is free; the other is clamped to a cover slip via the axoneme from which it is nucleated. These conditions dictate the appropriate set of $\mathcal{W}_{q}$. Using typical material parameters for microtubules $\left(L \sim \mu \mathrm{m}, \zeta_{\perp} \sim \mathrm{cP}, L_{p} \equiv A / k_{B} T \sim \mathrm{mm}\right)$, we observe from (12) that beyond the initial few milliseconds the shape is well approximated by a single mode, which decays with time constant $\tau_{1}=1 / \tilde{\nu} q_{1}^{4}$; here $q_{1} \simeq 1.875 / L$ is the smallest root of the clamped-free solvability condition $\cos (q L) \cosh (q L)=-1$. Measurement of $\tau_{1}$ thus gives $A$. The determination of the solvability condition generalizes to include pointlike forces exerted (e.g., by an optical trap) along the length of the biopolymer. The solutions to these equations, which are then functions of the location of the trap, may likewise be used to measure rigidity via dynamics.

The parametrization $y(x)$ readily generalizes to describe nonplanar curves in a Monge-like gauge $\mathbf{r}(x)=$ $[y(x), z(x)]$; the equation of motion is quite similar for small deformations: $\mathbf{r}_{t}=-\tilde{\nu} \mathbf{r}_{x x x x}$. A less trivial observation is that Eq. (1) also governs the intrinsic dynamics of three-dimensional elastic filaments [20]. From the three curvatures $\boldsymbol{\Omega}$ of the material frame we may construct a complex curvature [21] $\psi=\left(\Omega_{1}+i \Omega_{2}\right) \exp \left(i \int^{s} \Omega_{3}\right)$ whose leading order dynamic is $\psi_{t}=-\tilde{\nu} \psi_{\text {ssss }}+\ldots$.
Furthermore, for free elastic rods (at whose ends $\psi=$ $\psi_{s}=0$ ), the natural complete basis for $\psi$ is simply the set of biharmonic eigenfunctions $\mathcal{W}_{q}(s)$ (described above) satisfying $\mathcal{W}=\mathcal{W}_{s}=0$ at boundaries. The analytical tools developed for planar elastohydrodynamics are robust, and may be applied to the dynamics and instabilities of three-dimensional and even twisted elastica, as will be elucidated elsewhere [22].

We thank A. Ott and D. X. Riveline for collaborations, S. Block, S. Childress, J. Kessler, P. Nelson, C. O'Hern, and especially T. Powers for insightful discussions, and W. Bialek for bringing [5] to our attention. This work was supported by an NSF Presidential Faculty Fellowship, DMR 96-96257 (REG). We dedicate this work to the memory of E. Purcell.

[1] S. Childress, Swimming and Flying in Nature (Cambridge University Press, Cambridge, England, 1981).

[2] E. M. Purcell, Am. J. Phys. 45, 3 (1977).

[3] Low Reynolds Number Flows, film (Encyclopædia Brittanica Educational Corporation, New York, 1966).

[4] Sir J. Lighthill, Mathematical Biofluiddynamics (SIAM, Philadelphia, 1975).

[5] K. E. Machin, J. Exp. Biol. 35, 796 (1958).

[6] W. Ludwig, Z. Vergl. Physiol. 13, 397 (1930).

[7] D. Riveline, C.H. Wiggins, R. E. Goldstein, and A. Ott, Phys. Rev. E 56, 1330 (1997).

[8] J. Keller and S. Rubinow, J. Fluid Mech. 75, 705 (1976).

[9] G. G. Stokes, Trans. Cambridge Philos. Soc. 9, 8 (1851).

[10] As is appropriate for a bead held in an optical trap [7].

[11] C. Wiggins, D. Riveline, R. Goldstein, and A. Ott, Biophys. J. 74, 1043 (1998).

[12] J. Käs, H. Strey, M. Baermann, and E. Sackmann, Europhys. Lett. 21, 865 (1993); A. Ott, M. Magnasco, A. Simon, and A. Libchaber, Phys. Rev. E 48, 1642 (1993).

[13] G. I. Taylor, Proc. R. Soc. London A 211, 225 (1952).

[14] E. M. Purcell, Proc. Natl. Acad. Sci. U.S.A. 94, 11307 (1997).

[15] T. R. Strick, J.-F. Allemand, D. Bensimon, A. Bensimon, and V. Croquette, Science 271, 1835 (1996).

[16] We thank P. Nelson for this suggestion. See also C. Levinthal and H. R. Crane, Proc. Natl. Acad. Sci. U.S.A. 42, 436 (1956).

[17] See C. Brennan and H. Winet, Annu. Rev. Fluid Mech. 9, 339 (1997) for propulsion data on this and several other planar spermatazoa.

[18] L. Landau and L. Lifshitz, Theory of Elasticity (Pergamon, New York, 1986), p. 102; see also F. Gittes, B. Mickey, J. Nettleton, and J. Howard, J. Cell. Biol. 120, 923 (1993).

[19] H. Felgner, R. Frank, and M. Schliwa, J. Cell Sci. 109, 509 (1996).

[20] R. E. Goldstein and S. A. Langer, Phys. Rev. Lett. 75, 1094 (1995).

[21] H. Hasimoto, J. Fluid Mech. 51, 477 (1972); G. Darboux, Leçons sur la Théorie Genérale des Surfaces (GauthierVillars, Paris, 1915), Vol. I, p. 22.

[22] R.E. Goldstein, T. R. Powers, and C.H. Wiggins (to be published). 